Erratum

\title{
Erratum to "A Survey of FDG- and Amyloid-PET Imaging in Dementia and GRADE Analysis"
}

\author{
Daniela Perani, ${ }^{1}$ Orazio Schillaci, ${ }^{2}$ Alessandro Padovani, ${ }^{3}$ \\ Flavio Mariano Nobili, ${ }^{4}$ Leonardo Iaccarino, ${ }^{1}$ Pasquale Anthony Della Rosa, ${ }^{5}$ \\ Giovanni Frisoni, ${ }^{6,7}$ and Carlo Caltagirone ${ }^{8}$ \\ ${ }^{1}$ Nuclear Medicine Department, Vita-Salute San Raffaele University, San Raffaele Hospital and Division of Neuroscience, \\ San Raffaele Scientific Institute, Via Olgettina 60, 20132 Milan, Italy \\ ${ }^{2}$ Nuclear Medicine Department, University of Rome "Tor Vergata" and IRCCS Neuromed, 86077 Pozzilli, Italy \\ ${ }^{3}$ Department of Medical and Experimental Sciences, Unit of Neurology, Brescia University, 25123 Brescia, Italy \\ ${ }^{4}$ Department of Neuroscience Ophthalmology and Genetics, University of Genoa, 16132 Genoa, Italy \\ ${ }^{5}$ IBFM-CNR, Via Fratelli Cervi 93, Segrate, 20090 Milan, Italy \\ ${ }^{6}$ IRCCS Centro San Giovanni di Dio Fatebenefratelli, 25125 Brescia, Italy \\ ${ }^{7}$ Memory Clinic and LANVIE, Laboratory of Neuroimaging of Aging, University Hospitals and University of Geneva, \\ 1225 Geneva, Switzerland \\ ${ }^{8}$ University of Rome Tor Vergata and IRCCS S. Lucia, 00142 Rome, Italy
}

Correspondence should be addressed to Daniela Perani; perani.daniela@hsr.it

Received 12 May 2014; Accepted 22 May 2014; Published 11 June 2014

Copyright (C) 2014 Daniela Perani et al. This is an open access article distributed under the Creative Commons Attribution License, which permits unrestricted use, distribution, and reproduction in any medium, provided the original work is properly cited.

The authors' names were incorrectly listed as Perani Daniela, Schillaci Orazio, Padovani Alessandro, Nobili Flavio Mariano, Iaccarino Leonardo, Della Rosa Pasquale Anthony, Frisoni Giovanni, and Caltagirone Carlo; this error is corrected here.

The addresses have been corrected. 


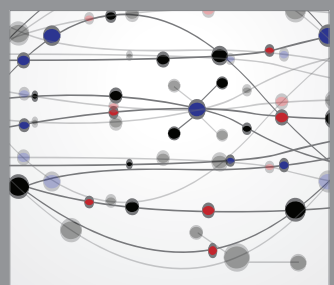

The Scientific World Journal
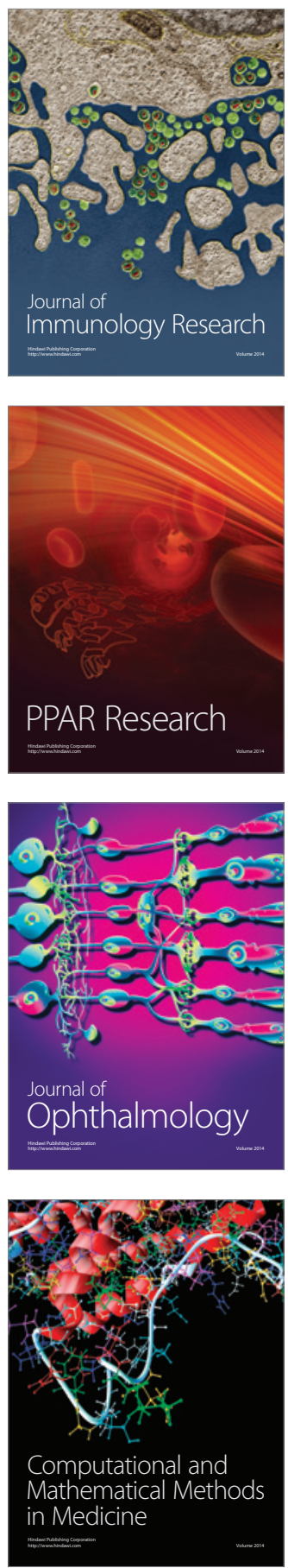

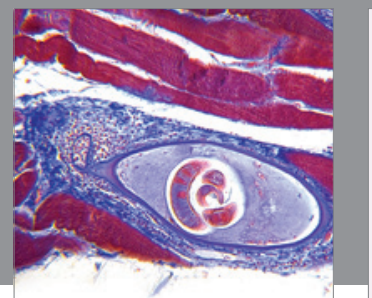

Gastroenterology

Research and Practice
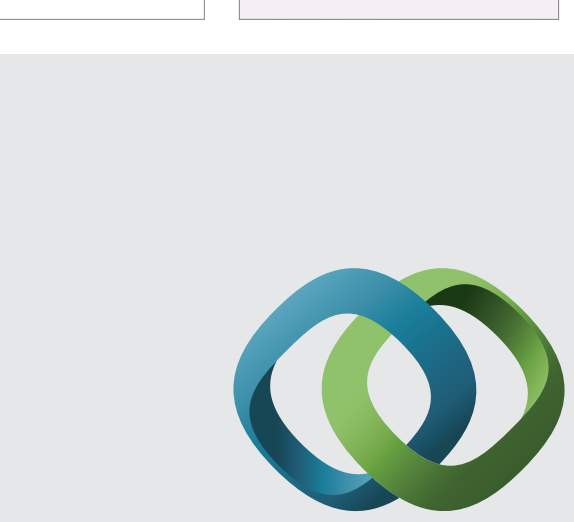

\section{Hindawi}

Submit your manuscripts at

http://www.hindawi.com
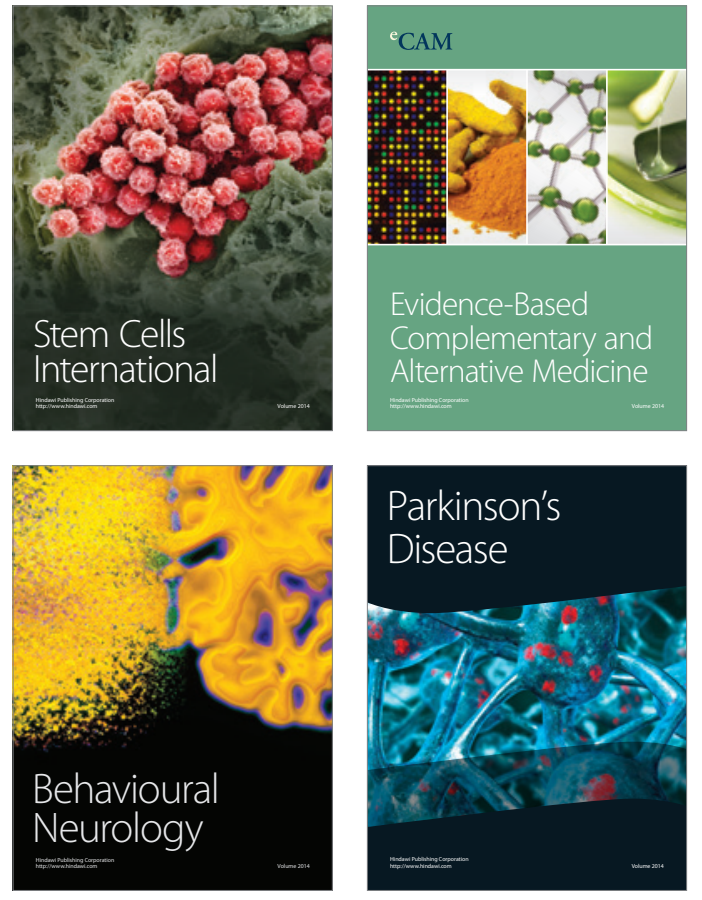
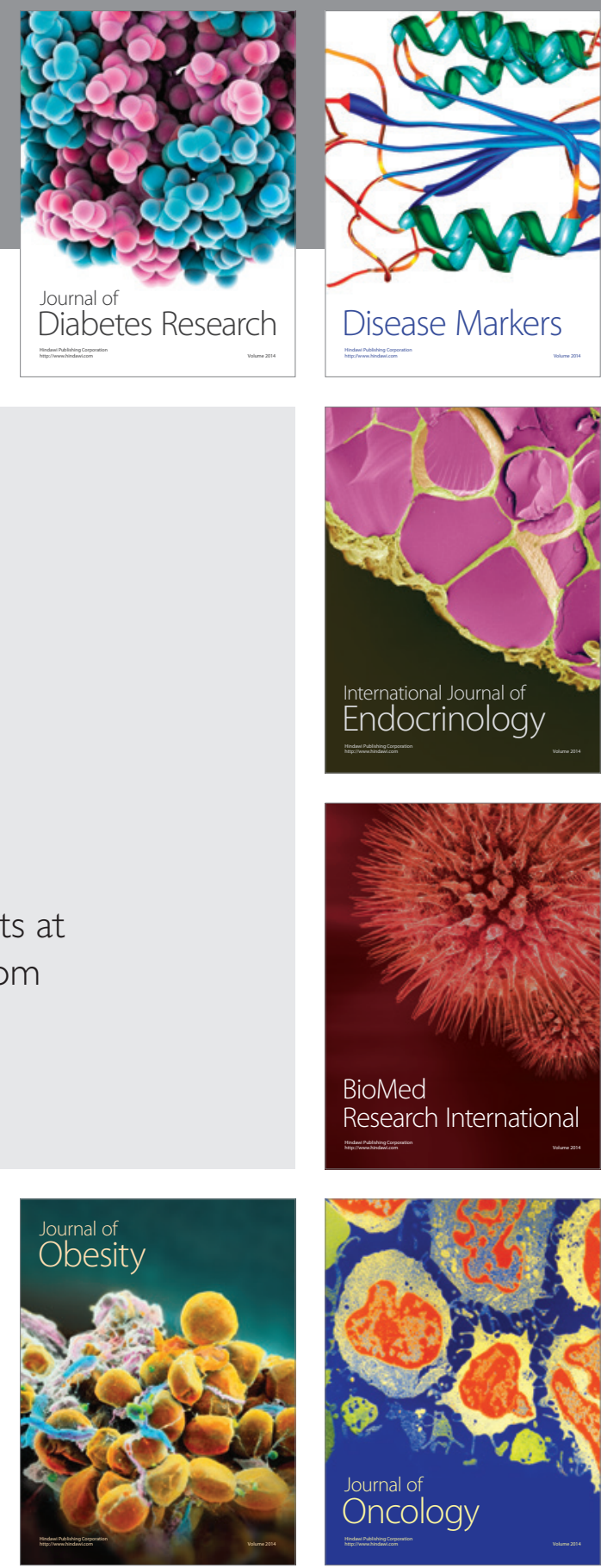

Disease Markers
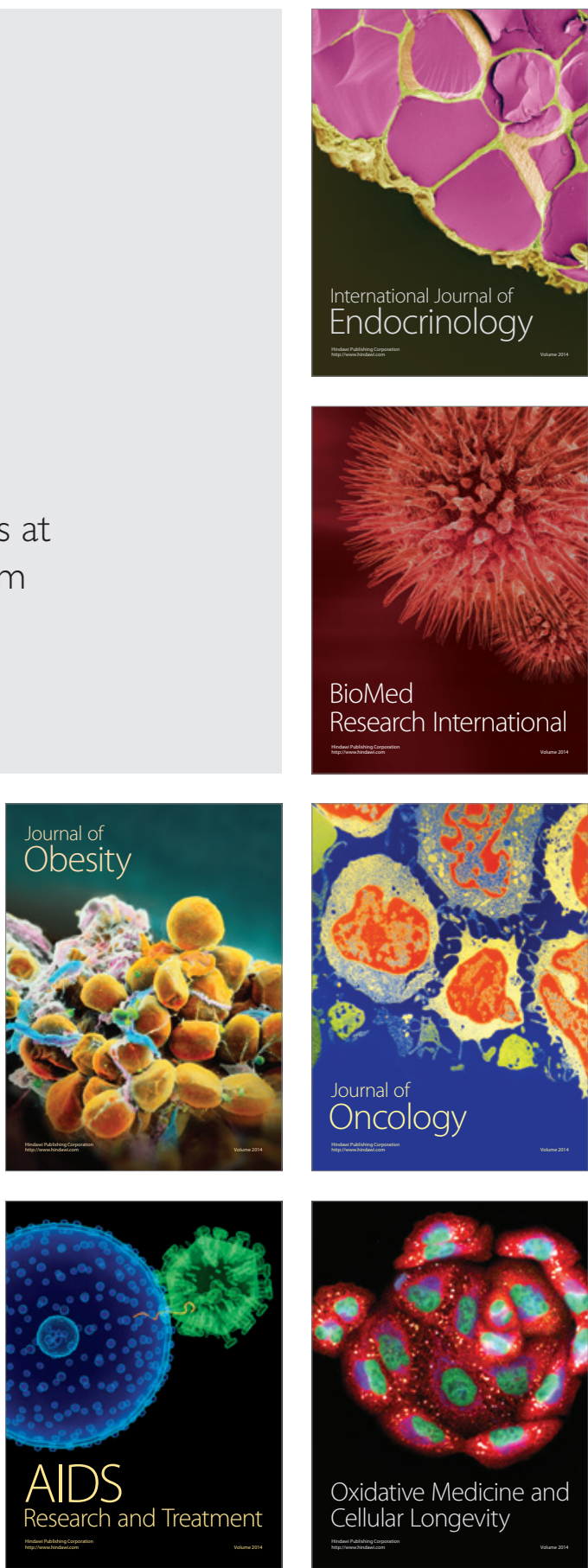